\title{
Ultrafast dynamics and coherent order parameter oscillations under photo-excitation in the excitonic insulator Ta2NiSe5
}

Daniel Werdehausen, Steinn Ymir Agustsson, Minjae Kim, Parmida Shabestari, Emily Huang, et al.

Daniel Werdehausen, Steinn Ymir Agustsson, Minjae Kim, Parmida Shabestari, Emily Huang, Amrit Pokharel, Timofei Larkin, Alexander Boris, Tomohiro Takayama, Yangfan Lu, Andreas W. Rost, Hao Chu, Alexander Yaresko, Marc Höppner, Armin Schulz, Dirk Manske, Bernhard Keimer, Hidenori Takagi, Stefan Kaiser, "Ultrafast dynamics and coherent order parameter oscillations under photo-excitation in the excitonic insulator Ta2NiSe5," Proc. SPIE 10638, Ultrafast Bandgap Photonics III, 1063803 (8 May 2018); doi: 10.1117/12.2304795 


\title{
Ultrafast dynamics and coherent order parameter oscillations under photo-excitation in the excitonic insulator $\mathrm{Ta}_{2} \mathrm{NiSe}_{5}$
}

\author{
Daniel Werdehausen ${ }^{\mathrm{a}, \mathrm{b}}$, Steinn Ymir Agustsson ${ }^{\mathrm{a}, \mathrm{b}}$, Minjae Kim ${ }^{\mathrm{a}, \mathrm{b}}$, Parmida Shabestari ${ }^{\mathrm{a}, \mathrm{b}}$, Emily \\ Huang $^{\mathrm{a}, \mathrm{b}}$, Amrit Pokharel ${ }^{\mathrm{a}, \mathrm{b}}$, Timofei Larkin ${ }^{\mathrm{a}}$, Alexander Boris ${ }^{\mathrm{a}}$, Tomohiro Takayama ${ }^{\mathrm{a}, \mathrm{c}}$, Yangfan \\ $\mathrm{Lu}^{\mathrm{d}}$, Andreas W. Rost ${ }^{\mathrm{a}, \mathrm{c}}$, Hao Chu ${ }^{\mathrm{a}, \mathrm{b}}$, Alexander Yaresko ${ }^{\mathrm{a}}$, Marc Höppner ${ }^{\mathrm{a}}$, Armin Schulz ${ }^{\mathrm{a}}$, Dirk \\ Manske $^{\mathrm{a}}$, Bernhard Keimer ${ }^{\mathrm{a}}$, Hidenori Takagi ${ }^{\mathrm{a}, \mathrm{c}, \mathrm{d}}$, Stefan Kaiser ${ }^{\mathrm{a}, \mathrm{b}^{*}}$ \\ ${ }^{a}$ Max-Planck-Institute for Solid State Research, Heisenbergstr. 1, 70569 Stuttgart, Germany; ${ }^{\mathrm{b}} 4$. \\ Physics Institute, University of Stuttgart, Pfaffenwaldring 57, 70569 Stuttgart, Germany; Institute \\ for Functional Matter and Quantum Technologies, University of Stuttgart, Pfaffenwaldring 57, \\ 70569 Stuttgart, Germany; ${ }^{\mathrm{d} D e p a r t m e n t}$ of Physics, University of Tokyo, Hongo 7-3-1. Bunkyo-ku, \\ Tokyo 113-003, Japan
}

\begin{abstract}
The excitonic insulator (EI) is an intriguing phase of condensed excitons undergoing a Bose-EinsteinCondensation (BEC)-type transition. A prominent candidate has been identified in $\mathrm{Ta}_{2} \mathrm{NiSe}_{5}$. Ultrafast spectroscopy allows tracing the coherent response of the EI condensate directly in the time domain. Probing the collective electronic response we can identify fingerprints for the Higgs-amplitude equivalent mode of the condensate. In addition we find a peculiar coupling of the EI phase to a low frequency phonon mode. We will discuss the transient response on multiple energies scales ranging from the exciton dynamics to the coherent $\mathrm{THz}$ response of the gap.
\end{abstract}

Keywords: excitonic insulator, exciton wave packet, collective excitation, ultrafast, pump-probe, non-equilibrium, timedomain, $\mathrm{THz}$

\section{INTRODUCTION}

\subsection{Excitonic insulators}

Excitonic insulators is a novel insulating state that is formed by the condensation of excitons (electron-hole pairs) into a common ground state. This state was theoretically predicted about 50 years ago to appear in small band gap semiconductors or semimetals with small band overlap, when the exciton binding energy exceeds either band gap or band width [1]. The condensation in these cases can be described either as a BEC or BCS-like transition for the two cases respectively.

A schematic picture is shown in Figure 1 describing the formation of an excitonic insulator gap. Excitons are bound states of electrons and holes due to their Coulomb interaction. In s simplified single-partice picture one can describe the bound excitons to appear below the bandgap of a semiconductor. However for large exciton binding energies exceeding the band gap of the semiconductor the exciton level would appear within the valence band of the semiconductor, as seen from the electron point of view, or inside the conduction band, as seen from the hole point of view. Therefore excitons form out of electrons and holes of the valence and conduction band that are within the exciton binding energy. At sufficient low temperatures these excitons then condense into a common ground state. This transition of preformed excitons that as bosons then condense into the excitonic insulator ground state at low temperatures can be described within a BEC-like picture. In the case of semimetals a similar picture emerges. Typically, in metals the background of quasi-free charge carriers screens the Coulomb interaction between electron hole excitations. Therefore no stable excitons form. However if the exciton binding energy exceeds the band overlap, e.g. in a semimetal, then the Coulomb interaction between the electrons and holes cannot be screened anymore. In this case excitons can form and they directly condense into the excitonic insulator ground state. This type of transition can be described within a BCS-like picture.

*s.kaiser@fkf.mpg.de; phone +49 711 689-1775; fax +49 711 689-1010; fkf.mpg.de/Kaiser

Ultrafast Bandgap Photonics III, edited by Michael K. Rafailov, Proc. of SPIE Vol. 10638, $1063803 \cdot$ C 2018 SPIE · CCC code: 0277-786X/18/\$18 · doi: 10.1117/12.2304795 
In contrast to superconductors no superfluid density, and accordingly no Meissner effect, appears because the condensed excitons are charge neutral. In optics, instead of the spectral weight being transferred into the zero frequency delta peak here the spectral weight gets transferred into an "exciton peak" at gap as shown in Figure 4.

Semimetal

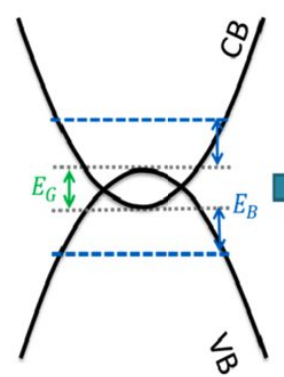

Excitonic Insulator

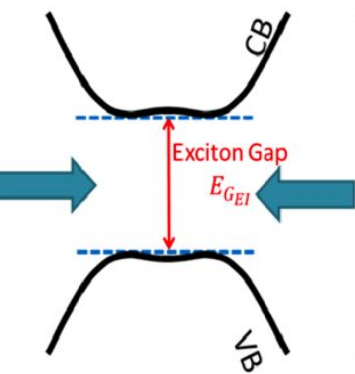

Semiconductor

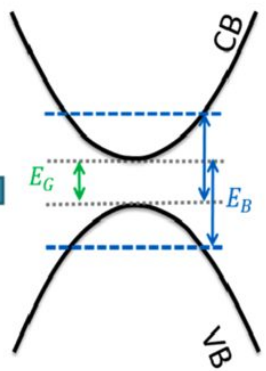

Figure 1: Formation of the excitonic gap in the excitonic insulator. In a semiconductor (right) the exciton binding energy $\mathrm{E}_{\mathrm{B}}$ exceeds the band gap $\mathrm{E}_{\mathrm{G}}$. In a simplified single-particle-like picture, as seen from the electron point of view, the exciton level (blue dashed line) below the conduction band appears to be inside the valence band. Respectively, seen from the hole point of view, the exciton levels appears in the conduction band. The electrons and holes in this energy range preform excitons and, at sufficient low temperatures, gap out the band structure forming a characteristic band flattening by condensing into the excitonic insulator state (middle). For a semimetal a similar transition appears when the screening of the Coulomb interaction breaks down.

\subsection{The excitonic insulator $\mathrm{Ta}_{2} \mathrm{NiSe}_{5}$}

Even though the existence of the excitonic insulator was first proposed in the 1960s [1-4] the unambiguous experimental identification of a material as an excitonic insulator still remains elusive. Two prominent compounds are $1 \mathrm{~T}-\mathrm{TiSe}_{2}$ and $\mathrm{TmSe}_{0.45} \mathrm{Te}_{0.55}$. The layered transition-metal dichalcogenide 1T- $\mathrm{TiSe}_{2}$ has an indirect band gap and therefore undergoes a transition into a charge density wave state at $200 \mathrm{~K}$ [5], which is accompanied by the formation of excitons [6]. In contrast the band gap of $\mathrm{TmSe}_{0.45} \mathrm{Te}_{0.55}$ can be tuned by applying pressure. Around 8 kbar a pronounced peak in the resistivity occurs, which might be caused by the formation of the excitonic insulator phase [7]. Another candidate that was discussed only recently in respect to the excitonic insulator phase is the chalcogenide compound $\mathrm{Ta}_{2} \mathrm{NiSe}_{5}[8,9]$ which today has become one of the most promising candidates for a purely electronic excitonic insulator [10].

$\mathrm{Ta}_{2} \mathrm{NiSe}_{5}$ is a quasi-1D layered compound, whose structure consist of $\mathrm{Ni}$ and Ta chains, which are aligned along the a axis of the crystal as shown in Figure 2. Along the b axis the layers are loosely held together by van der Waals' forces [11]. Resistivity measurements have shown that at high temperatures the material behaves like a small band gap semiconductor. At $\mathrm{T}_{\mathrm{C}}=328 \mathrm{~K}$, however, an anomaly in the resistivity occurs and the system transitions into a more insulating state [10]. This anomaly is accompanied by a change in the crystal structure from an orthorhombic to a monoclinic crystal system but without the formation of a CDW [12]. ARPES measurements revealed that this transition is accompanied by a band flattening, as it is characteristic for the excitonic insulator transition (see Figure 1) $[8,9]$.

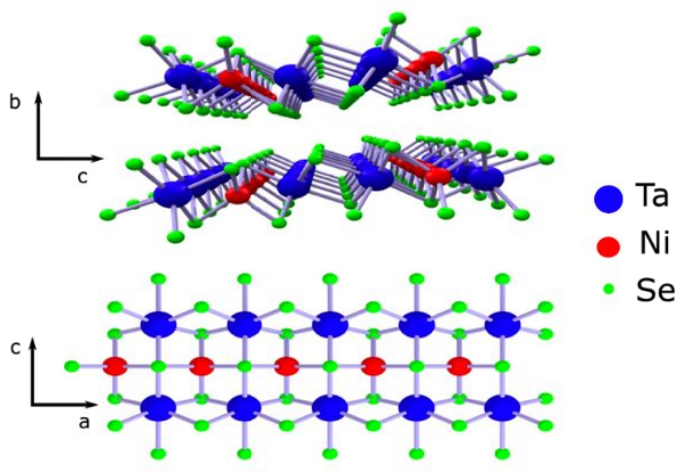

Figure 2: Structure of $\mathrm{Ta}_{2} \mathrm{NiSe}_{5}$. The material is made up of $\mathrm{Ni}$ and Ta chains, which run along the a axis of the crystal. 
Excitons in the Ta2NiSe5 are formed by a charge transfer between the Ni and Ta chains as sketched in Figure 3a. This real space separation is believed to be a reason to prevent a fast decay of electron-hole excitations and therefore stabilize the formation of excitons and open the possibility to condensate:
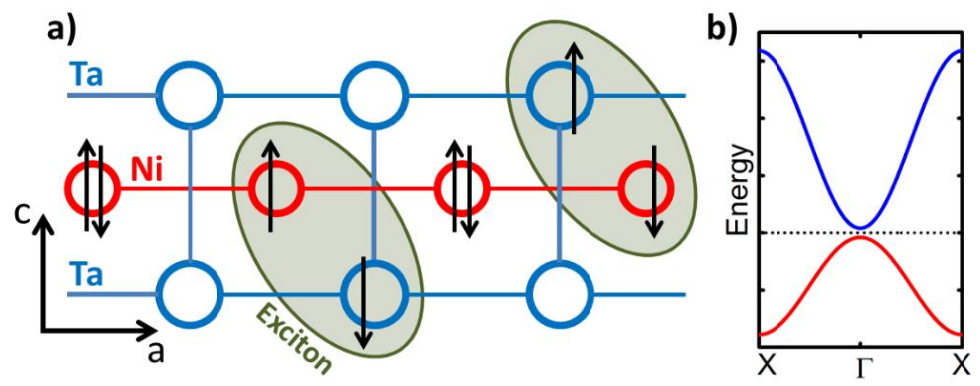

Figure 3: Exciton formation in real space (a) and band structure of Ta2NiSe5 (b). The material is a direct semiconductor with a very small band gap. The valance band is supplied by the Ni chain and the two degenerate conduction bands by the Ta chains. In real space an exciton is formed between an electron on the Ta chains and a hole on the Ni chain (a).

Band calculations revealed that the band structure around the Fermi level is rather simple [12]. The system is a direct semiconductor with a very small band gap, in which the valance band maximum and the conduction band minimum are located at the $\Gamma$ point (Figure $3 b$ ). The conduction band is doubly degenerate and the bands are quasi-1D and consequently have a cosine-like shape [12]. The conduction bands are supplied by the Ta chains (the $5 \mathrm{~d}_{\mathrm{xy}}$ orbitals) and the valence band is provided by the $\mathrm{Ni} 3 \mathrm{~d}_{\mathrm{xz}+\mathrm{yz}}$ orbitals, which hybridise with the $\mathrm{Se} 4 \mathrm{px}+\mathrm{y}$ orbitals [12]. This results in a spatial separation of the valance band from the conduction band.

\subsection{Optical properties of $\mathrm{Ta}_{2} \mathrm{NiSe}_{5}$}

Recent optical conductivity measurements confirmed that below $T_{C}$ an excitonic gap is opening, which reaches a size of $\Delta \mathrm{E} \approx 160 \mathrm{meV}$ at low temperatures $[10,13]$ as shown in Figure 4. On opening the gap the spectral weight is pushed into the "exciton peak" around gap. As detailed analysis of the gap and the exciton peak the panels on the right hand side of Figure 4 show the pronounced exciton resonances, which show that the formation of excitons is indeed driving the opening of the gap.

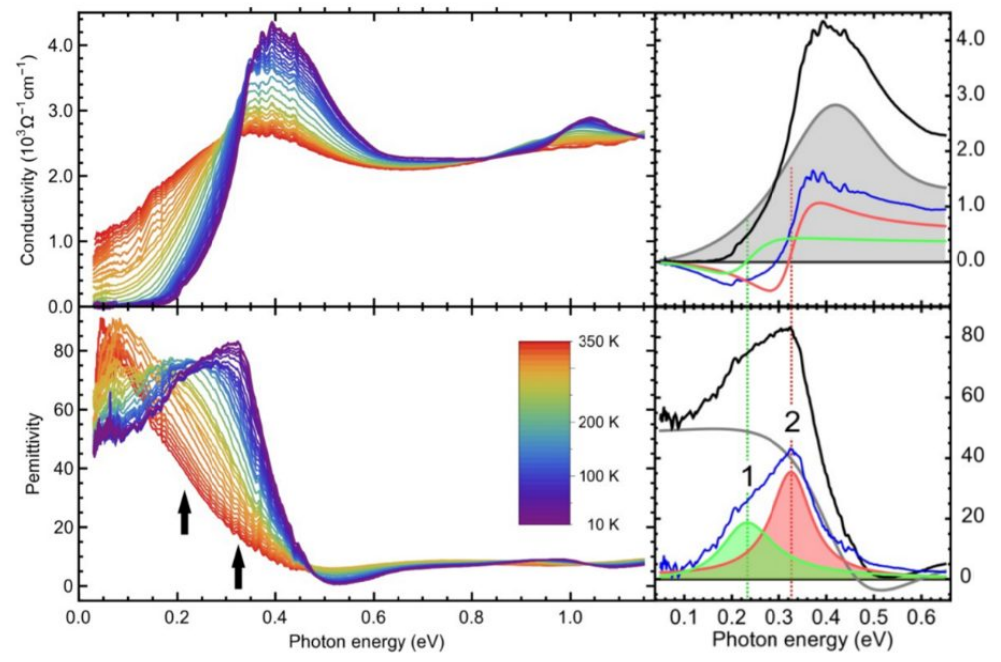

Figure 4: Optical conductivity and permittivity as function of photon energy obtained through ellipsometry [13]. The panels on the right show a generalized Lorentzian fits on the $10 \mathrm{~K}$ data revealing the electronic background (gray) and two Fano resonances corresponding to the exciton levels. 
The excitons lines appear as strong Fano resonances revealing spatially extended exciton-phonon states [13]. The extended complexes are key requirement for overlapping wavefunctions between the excitons and therefore the possibility to condense into an excitonic insulator ground state.

\section{ULTRAFAST PHOTO-EXCITATION}

However the optical measurements at equilibrium cannot directly probe any coherent properties of a potential excitonic insulator condensate. To this end ultrafast time domain measurements provide the unique opportunity to directly identify the symmetry broken state in the system. This can be achieved by probing the state's elementary collective excitations. The observation of the Higgs mode equivalent, i.e. a coherent amplitude oscillation of the order parameter, in a potential excitonic insulator would be direct evidence for the symmetry broken state [14].

\subsection{Melting and relaxation dynamics of the electronic system}

Ultrafast pump-probe measurements on $\mathrm{Ta}_{2} \mathrm{NiSe}_{5}$ have been performed to reveal the coherent response of the excionic insulator condensate. Strong ultra-short laser pulses at $1.55 \mathrm{eV}$ are used to excite the condensate. The high photon energy compared to the gap allows to partially depleting the ground state that subsequently recovers back into the equilibrium state on characteristic time scales. The dynamics of this process is probed with a time delayed second weaker pulse that probes the reflectivity changes. A typical dynamics is shown in Figure 5.

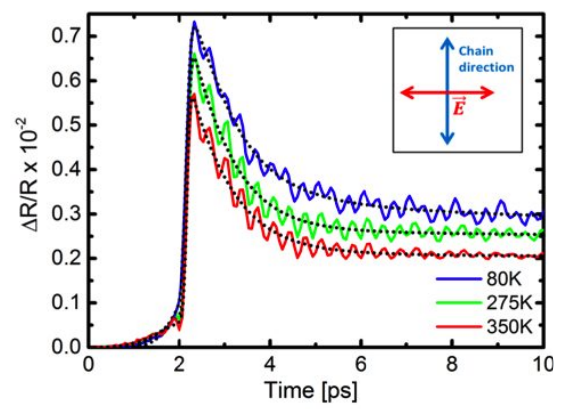

Figure 5: Time dependence of the photoinduced reflectivity changes in $\mathrm{Ta}_{2} \mathrm{NiSe}_{5}$. The inset illustrates the polarization of both the pump and the probe beams relative to the chains. The signal consists of an electronic component (black dotted lines) and several coherent oscillations.

On top of the electronic dynamics prominent coherent oscillations are present. These oscillations for one consist of coherent phonons but most spectacular also of a phonon coupled to the coherent excitonic insulator gound state, which we will discuss separately. Analyzing the melting and recovery times of the electronic quasiparticle dynamics as well as of the the non-coupled coherent phonon dynamics as a function of temperature and excitation fluence reveals that the material's intrinsic gap can be described as almost temperature independent for temperatures up to about $0.8^{*} \mathrm{~T}_{\mathrm{C}}$. This behavior supports the picture of the excitonic insulator state in $\mathrm{Ta}_{2} \mathrm{NiSe}_{5}$. An additional temperature dependent component above these temperatures suggests that the system is located in the BEC-BCS crossover regime [15].

\subsection{Coherent response of the electronic system}

To probe the systems cohenret electronic response is has to be excited and probed on its intrinsic time scales, i.e. on time scales of the excitonic gap. Such a response, measured with $\sim 11 \mathrm{fs}$ pulses, is shown in Figure 6. Very prominent oscillations are seen on the onset of the electronic signal. The spectrum reveals 3 prominent peaks at 30 and $34 \mathrm{THz}$ (red and green) as well as at $38 \mathrm{THz}$ (blue).

The first two peaks we can attribute to a exciton wavepacket beating. It results from a coherent beating of the different exciton levels against each other. In the time domain this is seen as oscillation at the difference frequency of the beating energy levels. Since the exciton levels $n=1$ and $n=2$ that are extracted from the equilibrium measurements (Figure 4 ) are at 210 and $330 \mathrm{meV}$, the expected beating signal between these excitons should appear equivalent to $120 \mathrm{meV}$, that is 30 
$\mathrm{THz}$, exactly where the first component is found (red). Assuming that to first order a Rydberg-like series holds for the excitons in the system one can derive an exciton binding energy of $E_{B}=4 / 3 * 120 \mathrm{meV}=160 \mathrm{meV}$. That is exactly the size of the optical gap. From that the expected beating between the $n=1$ and $n=3$ level would be at $8 / 9 * E_{B}=142 \mathrm{meV}$. That corresponds to $34.3 \mathrm{THz}$ in perfect agreement with the second mode (green) in the spectrum. Since our exciting laser pulse with $1.55 \mathrm{eV}$ is far above gap energy, we can exclude that the laser directly imprints the coherence to the beating exciton states. This suggests that the coherence might be intrinsic to the system, in agreement with the picture of a coherent excitonic state.
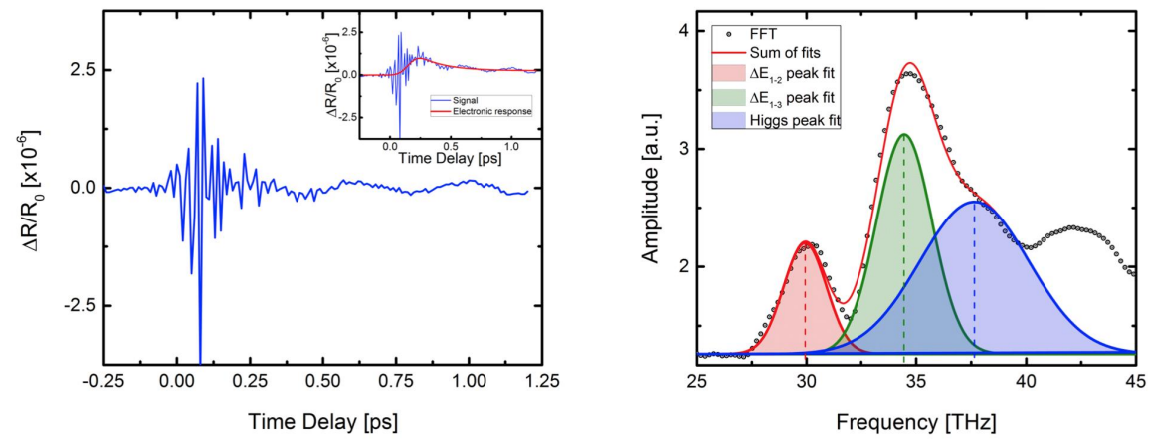

Figure 6: Left: High frequency oscillatory component of top of the change in reflectivity (inset). Right: Spectrum of the oscillations. We identified the first two (red and green) as quantum beating between excitons at the ground state ( $\mathrm{n}=1)$ and the first two excited states $(n=2, n=3)$. The blue peak we associate with possible Higgs-like oscialltions of the gap. The high frequency peak that is not fitted is due perturbed free induction decay of the ultrashort-pulse pump-probe experiment.

Further we have seen that the optical gap, as well as the extracted exciton binding energy, is about $160 \mathrm{meV}$. That corresponds to $38 \mathrm{THz}$, exactly where we find the $3^{\text {rd }}$ prominent contribution to the spectrum. A coherent oscillation directly at gap frequency could be interpreted as amplitude oscillation of the ground state and therefore as possible fingerprint for a Higgs-type order parameter oscillation of the excitonic insulator ground state.

\subsection{Coupled phonon-excitonic insulator order parameter oscillations}

For superconducting condensates it has been predicted recently that in the presence of strong electron phonon coupling, new collective modes can emerge, which show combined properties of the coherent phonon and electronic amplitude mode [16]. The strong Fano resonances in the optical spectra (Figure 4) are already a fingerprint for strong couplings to optical phonons in the system [13]. And indeed also a coupled phonon-excitonic insulator condensate mode has been identified [17]: A detailed temperature and fluence dependent study of the the coherent oscillations on top of the electronic response in Figure 5 reveals that the $1 \mathrm{THz}$ mode at high excitation fluences does not behave like a conventional coherent phonon but becomes a coupled phonon-excitonic insulator amplitude mode (Figure 7).
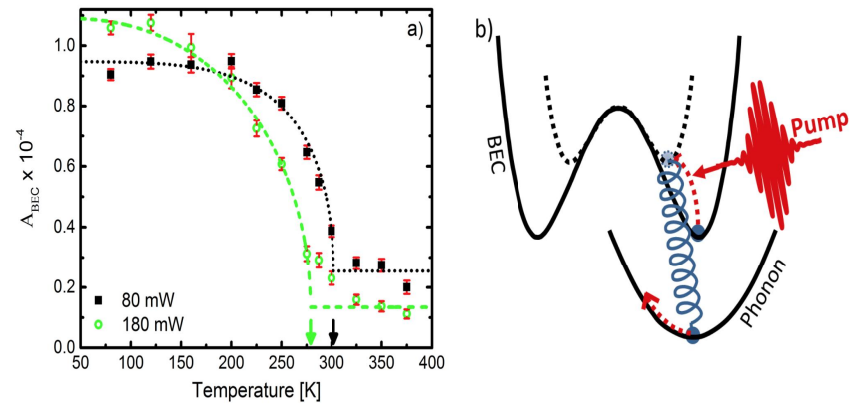

Figure 7: (a) Oscillation amplitude of the $1 \mathrm{THz}$ coupled phonon-excitonic insulator system. (b) Excitation mechanism: The pump pulse changes the condensed excitons potential energy landscape adiabatically by depleting the BEC of excitons. But this change occurs faster than the response time of the low frequency phonon, and therefore acts an impulsive excitation for the coupled phononcondensate system. 
Figure 7a shows the oscillation amplitudes of the $1 \mathrm{THz}$ mode that traces the temperature dependence of the transient order parameter for two different excitation fluences. A mean field type of behavior of the order parameter can describe these dependencies. The reduced $\mathrm{T}_{\mathrm{C}}$ for the higher excitation fluence is due to the larger depletion of the condensate.

\section{SUMMARY AND CONCLUSION}

The ultrafast dynamics of photo-excited $\mathrm{Ta}_{2} \mathrm{NiSe}_{5}$ reveals first direct fingerprints for a coherent dynamics of the excitons in the system, that are responsible for the opening of the gap. Furthermore strong exciton-phonon coupling allows the identification of a coupled phonon-excitonic insulator amplitude mode. This is a direct manifestation of order parameter oscillations in the excitonic condensate substantiating the existence of an excitonic insulator state in $\mathrm{Ta}_{2} \mathrm{NiSe}_{5}$.

\section{REFERENCES}

[1] Jerome, D., Rice, T. M., and Kohn, W., “ Excitonic Insulator” Phys. Rev. 158, 462 (1967).

[2] Mott, N., "The transition to the metallic state," Philosophical Magazine 6, 287 (1961).

[3] Knox, R. S., [Theory of excitons], vol. 5. Academic Press (1963).

[4] Keldysh, L. and Kopaev, Y. V., "Possible instability of semimetallic state toward coulomb interaction," Sov. Phys. Solid State, USSR 6, 2219 (1965).

[5] Porer, M., Leierseder, U., Menard, J.-M., Dachraoui, H., Mouchliadis, L., Perakis, I., Heinzmann, U., Demsar, J., Rossnagel, K., and Huber, R., "Non-thermal separation of electronic and structural orders in a persisting charge density wave," Nature materials 13, 857 (2014).

[6] Hellmann, S., Rohwer, T., Kallane, M., Hanff, K., Sohrt, C.,Stange, A., Carr, A., Murnane, M., Kapteyn, H., Kipp, L., et al., "Time-domain classification of charge-density-wave insulators," Nature communications 3, 1069 (2012).

[7] Bucher, B., Steiner, P., and Wachter, P., "Excitonic insulator phase in $\mathrm{TmSe}_{0.45} \mathrm{Te}_{0.55}$," Phys. Rev. Lett. 67, 2717 (1991).

[8] Wakisaka, Y., Sudayama, T., Takubo, K., Mizokawa, T., Arita, M., Namatame, H., Taniguchi, M., Katayama, N., Nohara, M., and Takagi, H., "Excitonic insulator state in $\mathrm{Ta}_{2} \mathrm{NiSe}_{5}$ probed by photoemission spectroscopy," Phys. Rev. Lett. 103, 026402 (2009).

[9] Seki, K., Wakisaka, Y., Kaneko, T., Toriyama, T., Konishi, T., Sudayama, T., Saini, N., Arita, M., Namatame, H., Taniguchi, M., et al., "Excitonic Bose-Einstein condensation in $\mathrm{Ta}_{2} \mathrm{NiSe}_{5}$ above room temperature," Phys. Rev. B 90, 155116 (2014).

[10]Lu, Y., Kono, H., Larkin, T., Rost, A., Takayama, T., Boris, A., Keimer, B., and Takagi, H., "Zero-gap semiconductor to excitonic insulator transition in Ta2NiSe5," Nature communication 8, 14408 (2017).

[11] Sunshine, S. A., and Ibers, J. A., "Structure and physical proper- ties of the new layered ternary chalcogenides tantalum nickel sulfide $\left(\mathrm{Ta}_{2} \mathrm{NiS}_{5}\right)$ and tantalum nickel selenide $\left(\mathrm{Ta}_{2} \mathrm{NiSe}_{5}\right)$," Inorg. Chemistry 24, 3611 (1985).

[12] Kaneko, T., Toriyama, T., Konishi, T., and Ohta, Y., "Orthorhombic-to-monoclinic phase transition of $\mathrm{Ta}_{2} \mathrm{NiSe}_{5}$ induced by the Bose-Einstein condensation of excitons," Phys. Rev. B 87, 035121 (2013).

[13] Larkin, T., Yaresko, A. , Propper, D. , Kikoin, K. , Takayama, T. , Mathis, Y.-L., Rost, A., Takagi, H., Keimer, B. and Boris, A., "Giant exciton Fano resonance in quasi-one dimensional $\mathrm{Ta}_{2} \mathrm{NiSe}_{5}$," Phys. Rev. B 95, 195144 (2017).

[14] Fauseweh, B., Schwarz, L., Tsuji, N., Cheng, N., Bittner, N., Krull, H., Berciu, M., Uhrig, G.S., Schnyder, A.P., Kaiser, S., Manske, D., "Higgs spectroscopy of superconductors in nonequilibrium", arxiv:1712.07989 (2017).

[15] Werdehausen, D., Takayama, T., Albrecht, G., Lu, Y., Takagi, H., and Kaiser, S., "Photo-excided Dynamics in the Excitonic Insulator $\mathrm{Ta}_{2} \mathrm{NiSe}_{5}$ ", arxiv:1801.10466 (2018).

[16] Murakami, Y., Werner, P., Tsuji, N., and Aoki, H., "Multiple amplitude modes in strongly coupled phononmediated superconductors," Phys. Rev. B 93, 094509 (2016).

[17] Werdehausen, D., Takayama, T., Höppner, M., Albrecht, G., Rost, A. W., Lu, Y., Manske, D., Takagi, H., and Kaiser, S., "Coherent Order Parameter Oscillations in the Ground State of the Excitonic Insulator $\mathrm{Ta}_{2} \mathrm{NiSe}_{5}$ ", Science Advances 4, eaap8652 (2018). 